\title{
Ratiometric Fluorescence Detection of 6-Mercaptopurine Based on the Nanohybrid of Fluorescence Carbon Dots and Gold Nanoclusters
}

\author{
Yanan Zhai ${ }^{1,2 *}$, Meixian Huang ${ }^{1,3^{*}}$, Lingfeng Jiang1,2\#, Hailin Liao ${ }^{2 \#}$ \\ ${ }^{1}$ Graduate School, Guangxi University of Chinese Medicine, Nanning, China \\ ${ }^{2}$ Guangxi Scientific Research Centre of Traditional Chinese Medicine, Guangxi University of Chinese Medicine, \\ Nanning, China \\ ${ }^{3}$ School of Pharmaceutical Sciences, Guangxi University of Chinese Medicine, Nanning, China \\ Email: "jianglingfeng@126.com, ${ }^{*}$ H-LLiao@126.com
}

How to cite this paper: Zhai, Y.N., Huang, M.X., Jiang, L.F. and Liao, H.L. (2021) Ratiometric Fluorescence Detection of 6Mercaptopurine Based on the Nanohybrid of Fluorescence Carbon Dots and Gold Nanoclusters. Journal of Sensor Technology, 11, 39-53.

https://doi.org/10.4236/jst.2021.113003

Received: September 2, 2021

Accepted: September 27, 2021

Published: September 30, 2021

Copyright $\odot 2021$ by author(s) and Scientific Research Publishing Inc. This work is licensed under the Creative Commons Attribution International License (CC BY 4.0).

http://creativecommons.org/licenses/by/4.0/

\section{(c) (i) Open Access}

\begin{abstract}
The development of a simple and accurate quantitative method for the determination of 6-mercaptopurine (6-MP) is of great importance because of its serious side effects. Ratiometric fluorescence (RF) sensors are not subject to interference from environmental factors, and exhibit enhanced precision and accuracy. Therefore, a novel RF sensor for the selective detection of 6-MP was developed. The present work reports a sensitive and selective RF sensor for the detection of 6-mercaptopurine, by hybridizing carbon nanodots (CDots) and gold nanoclusters (AuNCs) capped with bovine serum albumin (BSA). The CDots serve as the reference signal and the AuNCs as the reporter. On addition of the 6-MP, AuNCs formed aggregates, because the existing cross-links within the AuNCs and BSA structure were broken in favour of the Au-S bonds, which can enhance the fluorescence of AuNCs, while the fluorescence of CDots is stable against 6-MP, leading to distinct ratiometric fluorescence changes when exposed to 6-MP. 6-MP could be detected in the range of $0-30.22 \mu \mathrm{M}$ with a detection limit of $54 \mathrm{nM}$. The developed sensor was applied for the determination of 6-MP in human serum samples and satisfactory results were obtained.
\end{abstract}

\section{Keywords}

Gold Nanoclusters, Carbon Dots, 6-Mercaptopurine, Ratiometric Fluorescent Sensor 


\section{Introduction}

6-Mercaptopurine (6-MP), a sulfur analogue of adenine, is a kind of conventional chemotherapy anticancer drug [1], and is widely used in the therapy of acute lymphoblastic leukemia [2]. But as a cytotoxic anti-tumor drug, 6-MP always brings about serious side effects [3]. Moreover, the concentration of 6-MP in the plasma of a recipient varies, and it depends on individual differences [4] [5] [6]. The individual dosage regimens instead of standardized treatment regimens for some patients would make the drug concentration maintain at an optimal plasma level [4]. Therefore, it is still challenging to develop a simple and accurate quantitative method for 6-MP in order to monitor the concentrations of 6-MP in human serum.

Up to date, many techniques have been developed for the detection of 6-MP, including electrochemical (EC) methods [7] [8] [9] [10] [11], chemiluminescence (CL) [12] [13], high-performance liquid chromatography (HPLC) [14] [15], UV-vis spectrophotometry [16], and surface-enhanced Raman scattering spectroscopy [17] [18]. However, those methods suffer some limitations from reagents or the expensive equipment and specific sample pretreatment procedures. Therefore, an inexpensive, simple, sensitive, and accurate method for the detection of 6-MP is desired. Recently, development and use of fluorescent nanosensors (listed in Table 1) for the detection of 6-MP are the most widely reported [19]-[27].

In order to increase the selectivity and sensitivity, ratiometric fluorescent (RF) sensors are utilized, in which analyte concentrations are determined by measuring the ratios of the emission at two wavelengths [28]. Compared with singlechannel detection methods, RF sensors can avoid many problems, such as the drifts of the optoelectronic system (lamps and detectors), the probe concentration, autofluorescence in complicated biosystems, which are prone to disturbance in

Table 1. Comparison of published fluorescent nanosensors for the detection of 6-MP.

\begin{tabular}{llccc}
\hline \multicolumn{1}{c}{ Probe } & \multicolumn{1}{c}{ Detection principle } & $\begin{array}{c}\text { Detection } \\
\text { range }(\mu \mathrm{M})\end{array}$ & $\begin{array}{c}\text { Detection } \\
\text { limit }(\mu \mathrm{M})\end{array}$ & Refs. \\
\hline $\mathrm{MoS}_{2}$ quantum dots & Ratiometric fluorescence sensor & $0.5-70$ & 0.29 & {$[19]$} \\
$\mathrm{MOF}$ and quantum dots & Ratiometric fluorescence sensor & $0-50$ & 0.15 & {$[20]$} \\
Gold nanoparticles & Fluorescence enhancement & $0.0635-0.35$ & 0.000408 & {$[21]$} \\
Carbon dots & Fluorescence quenching & $0.04-12$ & 0.01 & {$[22]$} \\
Fe $\mathrm{O}_{4} @ S i \mathrm{O}_{2}$-AuNCs & Fluorescence decreasement & $0.01-0.5$ & 0.004 & {$[23]$} \\
Gold nanoparticles & Fluorescence switch sensor & $10-120$ & 0.000198 & {$[24]$} \\
CdTe quantum dots & Fluorescence quenching & $0.2-3.2$ & 0.08 & {$[25]$} \\
Nitrogen-doped carbon dots & Hybrid nano-sensors & $0.001-0.064$ & 0.00067 & {$[26]$} \\
MIP microspheres & MIP sensors & $0.0657-39.42$ & 0.0197 & {$[27]$} \\
CDots and AuNCs & Ratiometric fluorescence sensor & $0-30.22$ & 0.054 & This work \\
\hline
\end{tabular}


quantitative detection; therefore, they exhibit enhanced precision and accuracy [29]. Carbon nanodots (CDots) as a new type of biocompatible carbon-based nanomaterials have attracted tremendous attention because of their low toxicity, excellent water solubility, ease of synthesis and functionalization, and outstanding photostability [30]. Fluorescent gold nanoclusters (AuNCs) are emerging as novel fluorescent materials and have attracted more and more attention in the field of biolabeling, biosensing, bioimaging and targeted cancer treatment because of their unusual physicochemical properties, such as long fluorescence lifetime, ultrasmall size, large stokes shift, strong photoluminescence, as well as excellent biocompatibility and photostability [31]. The combined use of CDots and AuNCs together might suggest a new possibility to perform perfect fluorescence materials. Many researchers have built RF sensor based on CDots and AuNCs to detect Reactive Oxygen Species [32] [33], glucose [32], $\mathrm{Cd}^{2+}$ and L-ascorbic acid [34], hydrogen peroxide [35], $\mathrm{Hg}^{2+}$ [36] [37] [38], dopamine [39], cysteine [40].

In the present work, we have built a RF sensor for the detection of 6-MP by combining CDots and AuNCs. The CDots serve as the reference signal and the AuNCs as the reporter. On addition of the 6-MP, AuNCs formed aggregates, because the existing cross-links within the AuNCs and BSA structure were broken in favour of the thiol-Au bond, which can decrease the fluorescence of AuNCs, while the fluorescence of CDots is stable against 6-MP, leading to distinct ratiometric fluorescence changes when exposed to 6-MP. A limit of detection of 54 $\mathrm{nM}$ for 6-MP in aqueous solution was estimated. Thus, we applied the sensor for the detection of 6-MP in human serum.

\section{Material and Methods}

\subsection{Reagents and Instruments}

All chemicals were of analytical grade and used without further purification. Citric acid, ethylenediamine, sodium borohydride $\left(\mathrm{NaBH}_{4}\right)$, Chloroauric acid tetrahydrate $\left(\mathrm{HAuCl}_{4} \cdot 4 \mathrm{H}_{2} \mathrm{O}\right)$, bovine serum albumin (BSA), 6-mercaptopurine monohydrate, $\mathrm{NaH}_{2} \mathrm{PO}_{4}, \mathrm{Na}_{2} \mathrm{HPO}_{4}, \mathrm{NaCl}, \mathrm{KCl}, \mathrm{CaCl}_{2}, \mathrm{NH}_{3} \cdot \mathrm{H}_{2} \mathrm{O}, \mathrm{NaOH}, \mathrm{HCl}$, $\mathrm{MgSO}_{4}, \mathrm{Zn}\left(\mathrm{NO}_{3}\right)_{2}, \mathrm{Ni}\left(\mathrm{NO}_{3}\right)_{2}, \mathrm{Hg}\left(\mathrm{NO}_{3}\right)_{2}, \mathrm{CuSO}_{4}, \mathrm{Fe}\left(\mathrm{NO}_{3}\right)_{3}, \mathrm{Fe}\left(\mathrm{NO}_{3}\right)_{2}, \mathrm{~Pb}\left(\mathrm{NO}_{3}\right)_{2}$, $\mathrm{CdCl}_{2}, \mathrm{CrCl}_{3}$, Isolucine,, Uracil, Glucosuria, Aspartic acid, Tryptophan, Tyrosine, Lysine, Adenine, Cytosine, Cystine, Thiophenol, glutathione were purchased from Aladdin Biochemical Technology Co., Ltd. (Shanghai, China). All reagents were used as-received without additional purification. All the reagent solutions were prepared by the water purified through a Millipore system with a resistance of $18.2 \mathrm{M} \Omega \cdot \mathrm{cm}$.

Preparation for stock solution of 6-MP [25]. $\mathrm{N}_{2}$ was bubbled through an aqueous solution of $0.1 \mathrm{M} \mathrm{NaOH}$ until saturation to remove dissolved oxygen. $86 \mathrm{mg}$ 6-MP was added into $500 \mu \mathrm{L}$ of the aforementioned solution. $4.5 \mathrm{~mL}$ boiled ultrapure deionized water was added after the solid had dissolved completely. Then 100 mM 6-MP stock solution was prepared. The stock solution was diluted 
5 times and stored as $100 \mu \mathrm{L}$ per batch independent solution. The as-prepared solutions were stored in a $-20^{\circ} \mathrm{C}$ refrigerator. $1.90 \mathrm{~mL}$ boiled ultrapure deionized water per batch was added into the thawed solution before use.

The fluorescence spectra were recorded on an RF-6000 spectrofluorometer (Shimadzu, Japan) with $1 \mathrm{~cm}$ quartz cells. The light source used in the spectrofluorometer was a $150 \mathrm{~W}$ Xe arc lamp (Ushio Inc, Japan), and the emitted power density was approximately $20-32 \mathrm{~mW} \cdot \mathrm{cm}^{-2}$ in its wavelength range. The slits for excitation and emission monochromators width were both $5 \mathrm{~nm}$. The transmission electron microscopy (TEM) images were recorded using a JEOL 2010 transmission electron microscope. Fourier transform infrared (FT-IR) spectra were acquired from a Thermo Fisher Nicolet iS10 FT-IR spectrometer.

\subsection{Synthesis of Water-Soluble Cdots}

The CDots were prepared by a simple one-pot hydrothermal method with minor modifications [41]. Typically, $4.204 \mathrm{~g}$ of citric acid and $1.34 \mathrm{~mL}$ of 1,2-ethylenediamine were mixed and dissolved into $40 \mathrm{~mL}$ of water to form a clear solution. The mixture was put into a $50 \mathrm{~mL}$ poly(tetrafluoroethylene) Teflon-lined autoclave tube and the solution was sealed and treated at $200^{\circ} \mathrm{C}$ for $4 \mathrm{~h}$. The resulting brown solution was cooled to room temperature naturally and filtered through $0.45 \mu \mathrm{M}$ Suporfilters to remove the large or agglomerated particles. Then the CDots solution was purified by dialyzing against pure water using a membrane $\left(M_{\mathrm{W}}=3.5 \mathrm{kDa}\right)$ for $12 \mathrm{~h}$ and then storing at $4^{\circ} \mathrm{C}$ for further use.

\subsection{Synthesis of BSA-Au Nanoclusters}

All the glasswares were first washed with aqua regia and then rinsed with ultrapure water, several times before use. AuNCs were synthesized and purified according to the literature [42]. In a typical experiment, $\mathrm{HAuCl}_{4}$ solution $(10 \mathrm{~mL}$, $10 \mathrm{mM}$ ) was added to the BSA solution $(10 \mathrm{~mL}, 50 \mathrm{mg} / \mathrm{mL})$ under vigorous stirring. After $5 \mathrm{~min}$, suitable $\mathrm{NaOH}(1 \mathrm{M})$ solution was introduced to the mixture to adjust the $\mathrm{pH}$ to 11.0 and then the mixture was kept under stirring for $12 \mathrm{~h}$ at $37^{\circ} \mathrm{C}$. The solution color changed from pale yellow to brown. Then the resulting brown solution was purified by dialyzing against pure water using a membrane $\left(M_{\mathrm{W}}=12 \mathrm{kDa}\right)$ for $24 \mathrm{~h}$ and then storing at $4^{\circ} \mathrm{C}$ for further use.

\subsection{Determination of 6-MP Using the Nanohybrid Probe}

The nanohybrid system was prepared by the following procedure. First, in order to adjust the fluorescent intensity ratio of CDots and AuNCs to be 1:1, suitable CDots solution was added to $10 \mathrm{~mL}$ AuNCs solution. The mixture was kept under vigorous stirring for surface hybridization through reaction and interaction. The fluorescence spectra were recorded from 375 to $775 \mathrm{~nm}$ under excitation at $365 \mathrm{~nm}$. This nanohybrid probe shows a good physical stability [38].

To evaluate the sensitivity of the RF probe for the 6-MP, in $1.5 \mathrm{~mL}$ centrifuge tube, $100 \mu \mathrm{L}$ nanohybrid solution and $800 \mu \mathrm{L} \mathrm{PB}$ buffer $(\mathrm{pH} 8.0,50 \mathrm{mM})$ were 
added, then $100 \mu \mathrm{L}$ various concentrations of 6-MP were also added. The mixture was stirred. Subsequently, the fluorescence spectra were recorded from 375 to $775 \mathrm{~nm}$ under excitation at $365 \mathrm{~nm}$.

To further study the specificity of the sensing system towards detecting 6-MP, the interferences of co-existing foreign substances were tested under the aboveselected conditions, spiked with different substances of a known concentration individually.

\section{Results and Discussion}

\subsection{Design Strategy}

A schematic illustration of the ratiometric fluorescence bioassay platform for the detection of 6-MP based on the nanohybrid of fluorescence carbon dots and gold nanoclusters was shown in Figure 1. In brief, we first prepared the CDots and AuNCs. In the presence of 6-MP, the sulfhydryl group of 6-MP was preferentially bound with AuNCs through thiol-Au bond [23] and caused the fluorescence signal of AuNCs can be effectively quenched, whereas the fluorescence intensity of CDots is unaffected, which can serve as a better reference signal for 6-MP assay. By combining the two fluorescence behaviors, the nanohybrid represented an ideal platform for the ratiometric determination of 6-MP, with the AuNCs serving as the 6-MP recognition component and the CDots acting as the reference fluorophore.
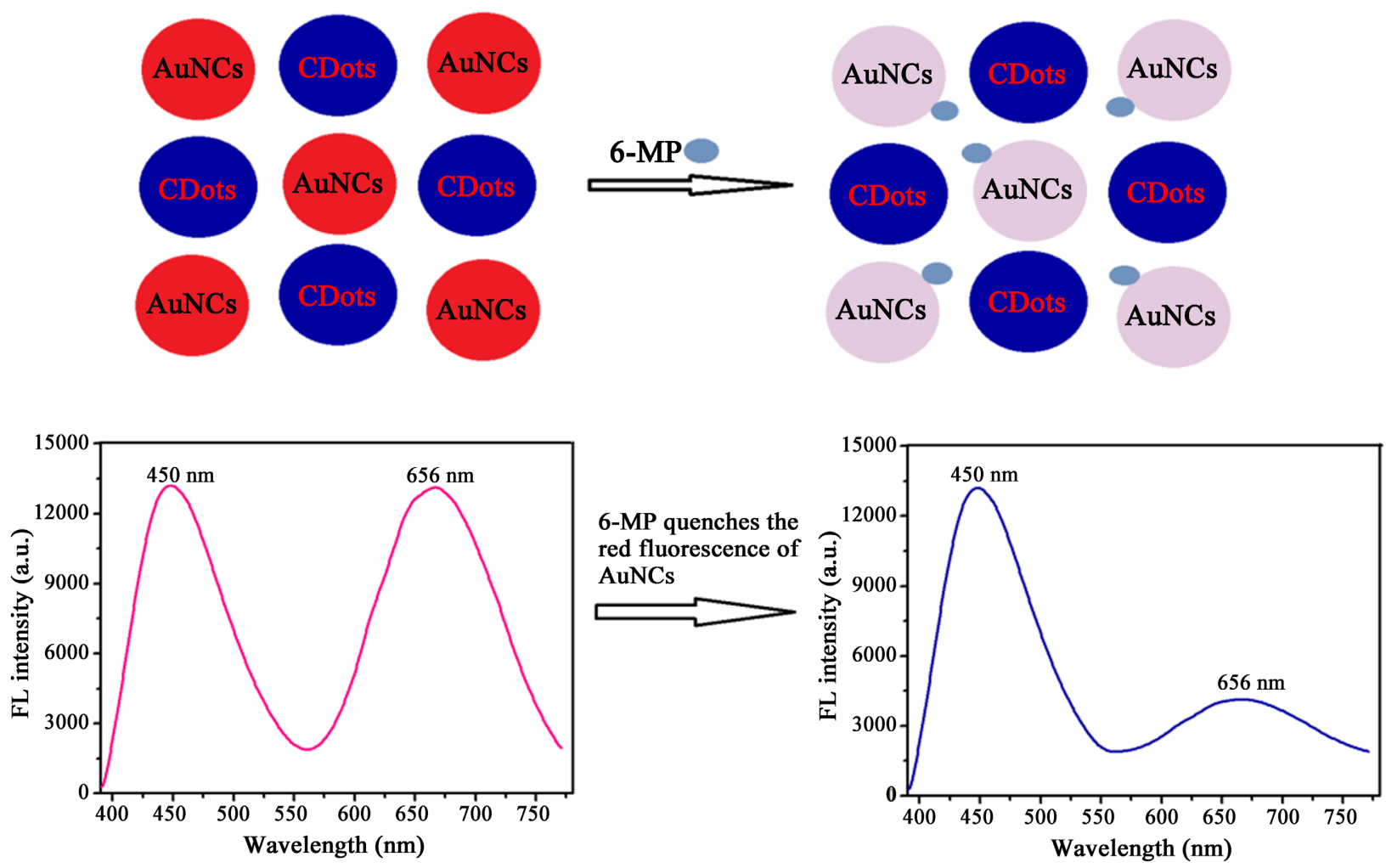

Figure 1. Schematic illustration of principle of 6-MP detection. The red fluorescence of AuNCs is quenched by 6-MP, while the blue fluorescence of CDots stay stable to 6-MP. 


\subsection{Characterization of the As-Prepared AuNCs and CDots}

The properties of the as-prepared Au NCs and CDots were investigated by fluorescence spectroscopy, TEM, and FT-IR spectra. Figure 2 shows the maximum emission center of AuNCs at $656 \mathrm{~nm}$ (Figure 2(a)), and the CDots emission band at $450 \mathrm{~nm}$ (Figure 2(b)). The fluorescence spectra of Au NCs and CDots were further recorded every $5 \mathrm{~min}$ for $1 \mathrm{~h}$ under ultraviolet irradiation at 365 $\mathrm{nm}$, and the fluorescence intensities exhibit no distinct change, implying that both the AuNCs and CDots exhibit good stability against photobleaching in aqueous solutions. The nanohybrid solution emits two emission bands at 450 and $656 \mathrm{~nm}$.

The morphologies and the sizes of the two components were characterized by TEM as shown in Figure 3 The diameter of CDots was estimated to be $\sim 4 \mathrm{~nm}$ with a good dispersity (Figure 3(a)). The AuNCs were readily dispersed in water and possessed a good monodispersity with a particle size of about $\sim 3 \mathrm{~nm}$ (Figure 3(b)). Additionally, CDots, AuNCs and CDots-AuNCs hybrid were characterized by FT-IR as shown in Figure 4 CDots have many characteristic peaks, such as $-\mathrm{OH}$ and $\mathrm{N}-\mathrm{H}\left(3100-3500 \mathrm{~cm}^{-1}\right), \mathrm{C}-\mathrm{H}\left(2930 \mathrm{~cm}^{-1}\right), \mathrm{C}=\mathrm{ONR}$

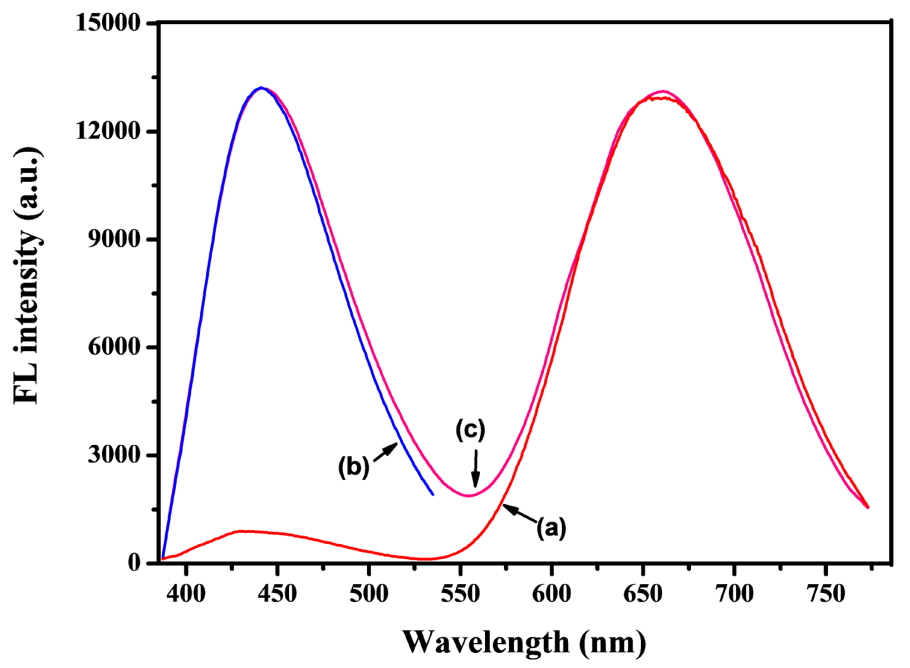

Figure 2. Fluorescence spectra of (a) emission of Au NCs, (b) emission of CDots and (c) the nanohybrid system.
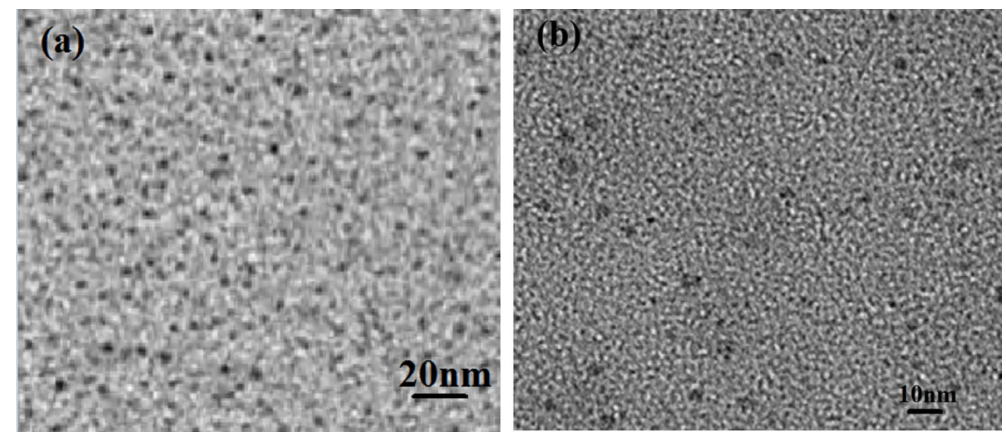

Figure 3. TEM images of the as-prepared (a) blue emission of CDots and (b) red emission of AuNCs. 


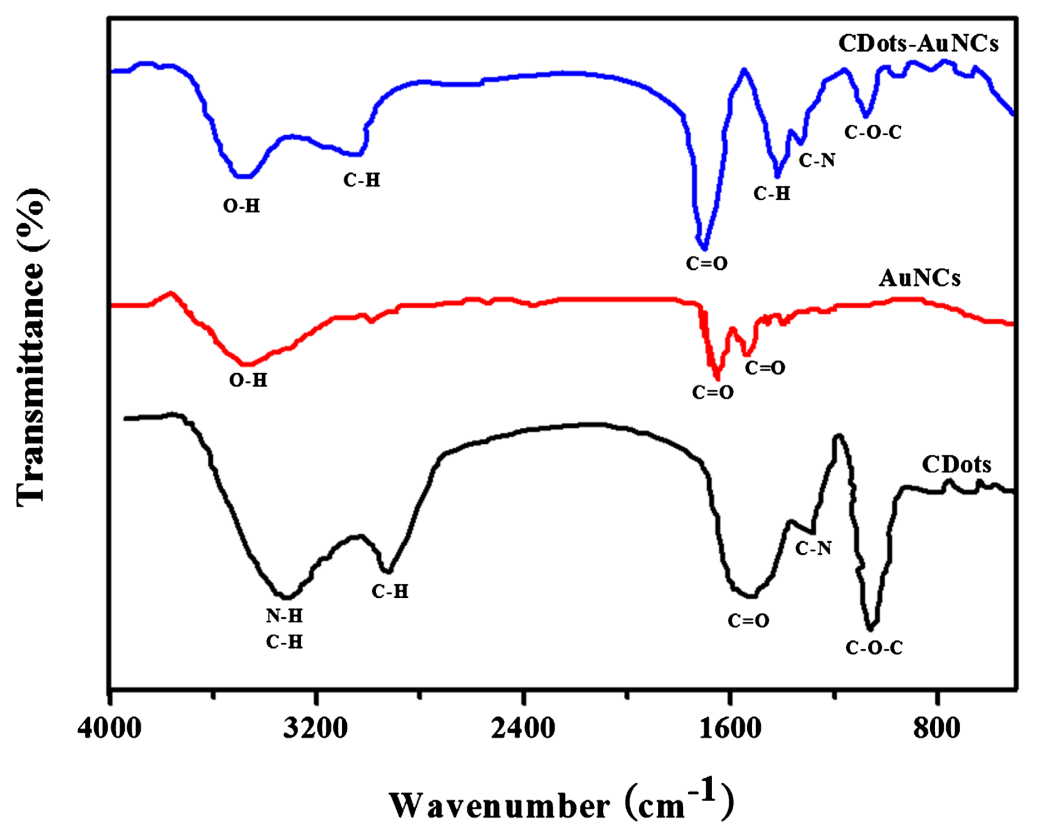

Figure 4. FT-IR spectrum of CDots, AuNCs and CDots-AuNCs hybrid.

(1640 $\left.\mathrm{cm}^{-1}\right), \mathrm{C}-\mathrm{N}\left(1290 \mathrm{~cm}^{-1}\right)$, and $\mathrm{C}-\mathrm{O}-\mathrm{C}\left(1084 \mathrm{~cm}^{-1}\right)$. The AuNCs showed stretching vibrations of $\mathrm{O}-\mathrm{H}$ at $3457 \mathrm{~cm}^{-1}, \mathrm{C}=\mathrm{O}$ stretching vibrations of carboxyl groups at 1661 and $1524 \mathrm{~cm}^{-1}$. For the CDots-AuNCs hybrid, three absorption bands at $3457 \mathrm{~cm}^{-1}, 1661$ and $1524 \mathrm{~cm}^{-1}$ were assigned to AuNCs, the characteristic peak at $1290 \mathrm{~cm}^{-1}$ could be assigned to the stretching vibration of the $\mathrm{C}-\mathrm{N}$ groups and $1084 \mathrm{~cm}^{-1}$ was related to $\mathrm{C}-\mathrm{O}$ stretching vibration from the CDots [39]. The results revealed that the CDots-AuNCs hybrid showed signals of both CDots and AuNCs.

\subsection{Establishment of Calibration Curve and Precision Measurement}

Figure 5 represents the fluorescence detection of 6-MP by the nanohybrid sensor. When the $I_{656} / I_{450}$ intensity ratio was adjusted to 1:1, as shown in Figure 3, the emission at $656 \mathrm{~nm}$ from the AuNCs gradually decreased upon the addition of 6-MP, but the fluorescence at $450 \mathrm{~nm}$ from the CDots was unchanged with the increase of 6-MP.

Figure 6 shows that the fluorescence intensity ratio, $I_{656} / I_{450}$ of the nanohybrid system decreased proportionately with increasing amounts of 6-MP, and a relationship can be set up between $I_{656} / I_{450}$ and the 6-MP concentration. The linear curve equation was $I_{656} / I_{450}=0.992-0.023 \times[6-\mathrm{MP}]$ with a correlation coefficient $R^{2}$ of 0.998 , indicating a good linear correlation between $I_{656} / I_{450}$ and the concentration of $6 \mathrm{MP}$. The detection limit for 6-MP was determined to be 54 $\mathrm{nM}$ based on the definition of 3 times deviation of the blank signal $(3 \sigma)$. The decline of the fluorescence ratio $\left(I_{656} / I_{450}\right)$ of the nanohybrid probe was attributed to the quenching of the fluorescence of the AuNCs by 6-MP. When the 6-MP was added, 6-MP was adsorbed on the BSA-AuNCs because the thiol-Au bond 


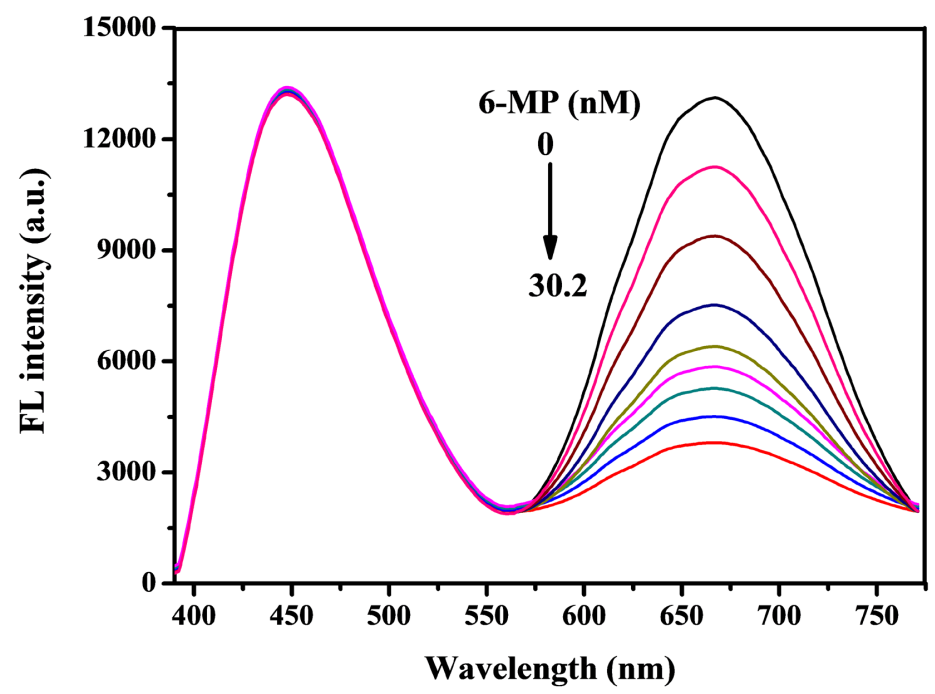

Figure 5. The fluorescence spectra of the nanohybrid system, in the presence of the 6-MP $(0.0,5.5,12.0,18.5,22.5,24.5,26.5,28.1,30.2 \mu \mathrm{M})$.

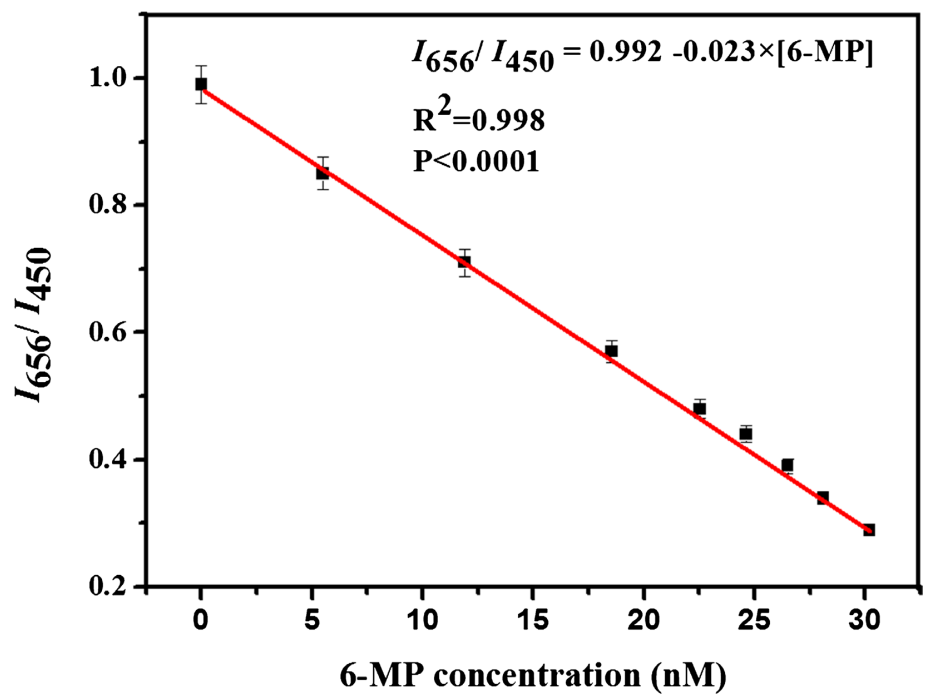

Figure 6. Fluorescence intensity ratio $\left(I_{656} / I_{450}\right)$ of the nanohybrid system versus the concentration of $6-\mathrm{MP}(0.00,5.51,12.01,18.52,22.53,24.52,26.53,28.11,30.22 \mu \mathrm{M})$. The detection limit for 6-MP was determined to be $54 \mathrm{nM}$.

can form. The formation of such bonds removed the protection effect of the BSA and neutralized the surface charge of the AuNCs. Without this protection effect of the BSA, the detached AuNCs coated with thiols, could aggregate, because there would be an increase in the van der Waals attraction forces between them [23].

In this study, precision was measured by intra-day and inter-day variability and expressed as the RSD, which was calculated from three replicate determinations of reference standard solution concentration of 6-MP at three concentrations $(5.5,22.5,30.2 \mu \mathrm{M})$ within one day (intra-day precision) and three replicates over three days (inter-day precision). The RSDs for the intra-day and inter-day precision were $2.3 \%-5.4 \%$ and $2.5 \%-6.8 \%$, respectively. 


\subsection{Optimization of the Experimental Conditions}

\subsubsection{Effect of Ion Strength}

Ion strength is always a key factor that influences a spectral method. In this work, we studied the effect of ion strength on detection of 6-MP using $\mathrm{NaCl}$ to regulate the ion intensity of the analytical system. The results were shown that the addition of $\mathrm{NaCl}$ strongly influences the analytical systems. So in the detection procedure, there is no need to add other electrolytes to adjust the ion strength of the detection system.

\subsubsection{Effect of $\mathrm{pH}$ Value}

The synthesis of AuNCs and in the present work was completed in a relative high $\mathrm{pH}$ environment. In order to study the effect of acidity on the ratiometric fluorescence of the analytical system, the effect of $\mathrm{pH}$ value was investigated from 4.5 to 10. During the work, small aliquots of $0.1 \mathrm{M} \mathrm{NaOH}$ or $\mathrm{HCl}$ was used to adjust the $\mathrm{pH}$ value. The results were shown that the fluorescence of nanohybrid probe strongly suffered by $\mathrm{pH}$. The fluorescence of nanohybrid probe was strongly decreased when the $\mathrm{pH}<7.0$, then almost unchanged from 7.5 to 10.0. Therefore, $\mathrm{pH}$ value 8.0 was determined as the optimal incubation $\mathrm{pH}$ value during the experiments.

\subsubsection{Dynamic Fluorescence Process of the Analytical System}

As to a spectral system, its stability affects its sensitivity and repeatability and thus we studied the time-dependent fluorescence of the analytical system by synchronous fluorescence with excitation at $365 \mathrm{~nm}$ and emission at $656 \mathrm{~nm}$. The results shown that the quenching effect of the 6-MP on the fluorescence intensity of AuNCs was quite fast in the first $10 \mathrm{~min}$, and the level of quenching remained unchanged for the next time; Therefore, the detection of 6-MP was measured after $10 \mathrm{~min}$.

\subsection{Selectivity of the Method for 6-MP Detection}

The interferences of co-existing foreign substances were tested under the aboveselected conditions, spiked with different substances of a known concentration individually. An error of $\pm 5 \%$ in the relative fluorescence intensity was considered tolerable. The results are summarized in Table 2. It can be seen from Table 2 that most of the tested substances including those of metal ions, some protein-forming amino acids, uracil, dextrose and glucosuria scarcely interfere with the determination at high tolerance levels. It is concluded that the method is free from many interferences of foreign substances. However, the thiol-containing compounds, cystine, thiophenol, glutathione, which were similar in structure to 6-MP, could be tolerated only at relative low levels. This suggested that the sensor responded differently with different thiol compounds. These compounds often have other different functional groups attached. Such as Yu et al. constructed a FRET assembly by using gold nanoclusters and carbon dots and their application as a ratiometric probe for cysteine [40]. Though AuNCs have been success- 
fully used in the determination of various metal ions such as $\mathrm{Hg}^{2+}, \mathrm{Fe}^{3+}, \mathrm{Cu}^{2+}$, $\mathrm{Pb}^{2+}$ and $\mathrm{Cr}^{3+}$ [34] [36] [37] [38] [43] [44] [45] [46], these metal ions did not affect the analytical results, the reason was that all these metal ions did not exist at alkaline solutions ( $\mathrm{pH}$ 8.0), they could form the corresponding precipitation of hydroxide with $\mathrm{OH}^{-}$. Thus, satisfactory analytical selectivity may be expected for different bio-thiols, and hence, the novel method has satisfactory selectivity for the analysis of 6-MP.

\subsection{Detection of 6-MP in Human Serum}

The proposed method was applied to the determination of 6-MP in spiked human serum. The serum samples, obtained from healthy volunteers, were spiked with 6-MP at different concentrations, and treated as the recommended procedure. The concentrations of 6-MP were calculated from the calibration graph. The results obtained for the determination of 6-MP in spiked human serum are given in Table 3 . The satisfactory recoveries obtained with such a simple sample

Table 2. Interferences of co-existing foreign substances.

\begin{tabular}{cccccc}
\hline $\begin{array}{c}\text { Foreign } \\
\text { substance }\end{array}$ & $\begin{array}{c}\text { Concentratio } \\
\mathbf{n}(\times 30.2 \mu \mathrm{M})\end{array}$ & $\begin{array}{c}\text { Change of } \\
I_{672} / I_{457}(\%)\end{array}$ & $\begin{array}{c}\text { Foreign } \\
\text { substance }\end{array}$ & $\begin{array}{c}\text { Concentratio } \\
\mathbf{n}(\times 30.2 \mu \mathrm{M})\end{array}$ & $\begin{array}{c}\text { Change of } \\
I_{672} / I_{457}(\%)\end{array}$ \\
\hline $\mathrm{Na}^{+}, \mathrm{Cl}^{-}$ & 50.0 & 2.53 & Isolucine & 10.0 & 3.77 \\
$\mathrm{~K}^{+}, \mathrm{Cl}^{-}$ & 50.0 & 1.25 & Uracil & 10.0 & 3.58 \\
$\mathrm{Ca}^{2+}, \mathrm{Cl}^{-}$ & 50.0 & -1.95 & Glucosuria & 10.0 & 1.98 \\
$\mathrm{Mg}^{2+}, \mathrm{SO}_{4}^{2-}$ & 50.0 & 3.85 & Aspartic acid & 10.0 & 2.56 \\
$\mathrm{Zn}^{2+}, \mathrm{NO}_{3}^{-}$ & 10.0 & -3.81 & Tryptophan & 10.0 & 2.57 \\
$\mathrm{Ni}^{2+}, \mathrm{NO}_{3}^{-}$ & 10.0 & 4.52 & Tyrosine & 10.0 & 2.39 \\
$\mathrm{Hg}^{2+}, \mathrm{NO}_{3}^{-}$ & 10.0 & -4.32 & Lysine & 10.0 & 3.52 \\
$\mathrm{Cu}^{2+}, \mathrm{SO}_{4}^{2-}$ & 10.0 & -4.06 & Adenine & 10.0 & 2.65 \\
$\mathrm{Fe}^{3+}, \mathrm{NO}_{3}^{-}$ & 10.0 & -4.53 & Cytosine & 10.0 & 1.95 \\
$\mathrm{Fe}^{2+}, \mathrm{NO}_{3}^{-}$ & 10.0 & 3.52 & Glycine & 10.0 & 1.95 \\
$\mathrm{Cd}^{2+}, \mathrm{Cl}^{-}$ & 10.0 & 2.35 & Cystine & 0.05 & -4.99 \\
$\mathrm{Cr}^{3+}, \mathrm{Cl}^{-}$ & 10.0 & 4.21 & Thiophenol & 0.05 & -0.88 \\
$\mathrm{~Pb}^{2+}, \mathrm{NO}_{3}^{-}$ & 10.0 & 4.15 & glutathione & 0.05 & -4.86 \\
\hline
\end{tabular}

Table 3. Determination of 6-MP in spiked human serum.

\begin{tabular}{ccccc}
\hline Samples & 6-MP spiked $(\mu \mathrm{M})$ & 6-MP founded $(\mu \mathrm{M})^{\mathrm{a}}$ & Recovery $(\%)$ & R.S.D. $(\%)^{\mathrm{b}}$ \\
\hline 1 & 0.00 & 0.00 & - & - \\
2 & 5.51 & 5.49 & 99.6 & 1.92 \\
3 & 24.52 & 24.38 & 99.4 & 2.01 \\
4 & 30.22 & 30.39 & 100.6 & 1.85 \\
\hline
\end{tabular}

a Mean values of 11 determinations. ${ }^{b}$ Relative standard deviation. 
procedure are in the range of $99.6 \%-101.6 \%$.

\section{Conclusion}

In conclusion, we designed a ratiometric fluorescence probe by hybridizing the CDots and the AuNCs. The nanohybrid probe exhibited dual emissions at 450 and $656 \mathrm{~nm}$ under a single excitation. The fluorescence at $450 \mathrm{~nm}$ was inert to 6-MP, while the fluorescence at $656 \mathrm{~nm}$ showed good specificity to 6-MP, leading to distinct ratiometric fluorescence changes. 6-MP could be detected in the range of $0-30.22 \mu \mathrm{M}$ with a detection limit of $54 \mathrm{nM}$. The proposed method was satisfactorily applied for analysis of 6-MP in human serum.

\section{Acknowledgements}

This work was supported by Self-Topic Fund of Guangxi Key Laboratory of Chinese Medicine Foundation Research, Guangxi University of Chinese Medicine, China (No. 19-050-45-05), and Open Project of 2019-2021 Guangxi First-class Discipline Construction, Guangxi University of Chinese Medicine, China (No. 2019XK131).

\section{Conflicts of Interest}

The authors declare no conflicts of interest regarding the publication of this paper.

\section{References}

[1] Sahasranaman, S., Howard, D. and Roy, S. (2008) Clinical Pharmacology and Pharmacogenetics of Thiopurines. European Journal of Clinical Pharmacology, 64, 753-767. https://doi.org/10.1007/s00228-008-0478-6

[2] Nielsen, O.H., Vainer, B. and Rask-Madsen, J. (2001) Review Article: The Treatment of Inflammatory Bowel Disease with 6-Mercaptopurine or Azathioprine. Alimentary Pharmacology \& Therapeutics, 15, 1699-1708.

https://doi.org/10.1046/j.1365-2036.2001.01102.x

[3] Kanemitsu, H., Yamauchi, H., Komatsu, M., Yamamoto, S., Okazaki, S., Uchida, K. and Nakayama, H. (2009) 6-Mercaptopurine (6-MP) Induces Cell Cycle Arrest and Apoptosis of Neural Progenitor Cells in the Developing Fetal Rat Brain. Neurotoxicology and Teratology, 31, 104-109. https://doi.org/10.1016/j.ntt.2008.10.001

[4] Su, Y., Hen, Y.Y., Chu, Y.Q., Van de Poll, M.E.C. and Relling, M.V. (1999) Assay of 6-Mercaptopurine and Its Metabolites in Patient Plasma by High-Performance Liquid Chromatography with Diode-Array Detection. Journal of Chromatography B: Biomedical Sciences and Applications, 732, 459-468. https://doi.org/10.1016/S0378-4347(99)00311-4

[5] Zimm, S., Collins, J.M., Riccardi, R., O’Neill, D., Narang, P.K., Chabner, B. and Poplack, D.G. (1983) Variable Bioavailability of Oral Mercaptopurine Is Maintenance Chemotherapy in Acute Lymphoblastic Leukemia Being Optimally Delivered. The New England Journal of Medicine, 308, 1005-1009. https://doi.org/10.1056/NEJM198304283081705

[6] Jacqz-Aigrain, E., Nafa, S., Médard, Y., Bessa, E., Lescoeur, B. and Vilmer, E. (1997) 
Pharmacokinetics and Distribution of 6-Mercaptopurine Administered Intravenously in Children with Lymphoblastic Leukaemia. European Journal of Clinical Pharmacology, 53, 71-74. https://doi.org/10.1007/s002280050339

[7] Vinita, T.M., Agnihotri, N., Singh, M., Singh, A.K. and Prakash, R. (2019) Nanonetwork of Coordination Polymer AHMT-Ag for the Effective and Broad Spectrum Detection of 6-Mercaptopurine in Urine and Blood Serum. ACS Omega, 4, 16733 16742. https://doi.org/10.1021/acsomega.9b01122

[8] Kumar, A., Pathak, P.K. and Prasad, B.B. (2019) Electrocatalytic Imprinted Polymer of N-Doped Hollow Carbon Nanosphere-Palladium Nanocomposite for Ultratrace Detection of Anticancer Drug 6-Mercaptopurine. ACS Applied Materials \& Interfaces, 11, 16065-16074. https://doi.org/10.1021/acsami.9b02947

[9] Hanko, M., Svorc, L., Plankova, A. and Mikus, P. (2019) Novel Electrochemical Strategy for Determination of 6-Mercaptopurine Using Anodically Pretreated Boron-Doped Diamond Electrode. Journal of Electroanalytical Chemistry, 840, 295 304. https://doi.org/10.1016/j.jelechem.2019.03.067

[10] Hatamluyi, B. and Es'haghi, Z. (2018) Electrochemical Biosensing Platform Based on Molecularly Imprinted Polymer Reinforced by ZnO-Graphene Capped Quantum Dots for 6-Mercaptopurine Detection. Electrochimica Acta, 283, 1170-1177. https://doi.org/10.1016/j.electacta.2018.07.068

[11] Cao, X.N., Lin, L., Zhou, Y.Y., Shi, G.Y., Zhang, W., Yamamoto, K. and Jin, L.T. (2003) Amperometric Determination of 6-Mercaptopurine on Functionalized Multi-Wall Carbon Nanotubes Modified Electrode by Liquid Chromatography Coupled with Microdialysis and Its Application to Pharmacokinetics in Rabbit. Talanta, 60, 1063-1070. https://doi.org/10.1016/S0039-9140(03)00187-5

[12] Sun, H.W., Wang, T., Liu, X.Y. and Chen, P.Y. (2013) A Sensitive Inhibition Chemiluminescence Method for the Determination of 6-Mercaptopurine in Tablet and Biological Fluid Using the Reaction of Luminol-Ag(III) Complex in Alkaline Medium. Journal of Luminescence, 134, 154-159. https://doi.org/10.1016/j.jlumin.2012.08.056

[13] Biparva, P., Abedirad, S.M. and Kazemi, S.Y. (2015) Silver Nanoparticles Enhanced a Novel TCPO- $\mathrm{H}_{2} \mathrm{O}_{2}$-Safranin O Chemiluminescence System for Determination of 6-Mercaptopurine. Spectrochimica Acta Part A: Molecular and Biomolecular Spectroscopy, 145, 454-460. https://doi.org/10.1016/j.saa.2015.03.019

[14] Somasekhar, V. (2014) Optimization and Validation of an RP-HPLC Method for the Estimation of 6-Mercaptopurine in Bulk and Pharmaceutical Formulations. Brazilian Journal of Pharmaceutical Sciences, 50, 793-797.

https://doi.org/10.1590/S1984-82502014000400015

[15] Zakrzewski, R., Borowczyk, K., Luczak, A., Mlynarski, W. and Trelinska, J. (2013) Determination of Urinary 6-Mercaptopurine and Three of Its Metabolites by HPLC-UV Coupled with the Iodine-Azide Reaction. Bioanalysis, 5, 869-877. https://doi.org/10.4155/bio.13.46

[16] Diamai, S., Warjri, W., Saha, D. and Negi, D.P.S. (2018) Sensitive Determination of 6-Mercaptopurine Based on the Aggregation of Phenylalanine-Capped Gold Nanoparticles. Colloid Surface A, 538, 593-599.

https://doi.org/10.1016/j.colsurfa.2017.11.052

[17] Zhang, L.X., Li, H.L., Chu, G., Luo, L., Jin, J., Zhao, B. and Tian, Y. (2016) Detection of 6-Mercaptopurine by Silver Nanowires-Coated Silicon Wafer Based on SurfaceEnhanced Raman Scattering Spectroscopy. Colloid Surface A, 508, 309-315. https://doi.org/10.1016/j.colsurfa.2016.08.069 
[18] Yang, L., Chen, Y.H., Li, H.L., Luo, L., Zhao, Y., Zhang, H.Q. and Tian, Y. (2015) Application of Silver Nanoparticles Decorated with Beta-Cyclodextrin in Determination of 6-Mercaptopurine by Surface-Enhanced Raman Spectroscopy. Analytical Methods, 7, 6520-6527. https://doi.org/10.1039/C5AY01212K

[19] Zhang, F., Liu, H., Liu, Q. and Su, X.G. (2018) An Enzymatic Ratiometric Fluorescence Assay for 6-Mercaptopurine by Using $\mathrm{MoS}_{2}$ Quantum Dots. Microchimica Acta, 185, 540-547. https://doi.org/10.1007/s00604-018-3039-4

[20] Jin, M., Mou, Z.L., Zhang, R.L., Liang, S.S. and Zhang, Z.Q. (2017) An Efficient Ratiometric Fluorescence Sensor Based on Metal-Organic Frameworks and Quantum Dots for Highly Selective Detection of 6-Mercaptopurine. Biosensors and Bioelectronics, 91, 162-168. https://doi.org/10.1016/j.bios.2016.12.022

[21] Shen, X.C., Jiang, L.F., Liang, H., Lu, X., Zhang, L.J. and Liu, X.Y. (2006) Determination of 6-Mercaptopurine Based on the Fluorescence Enhancement of Au Nanoparticles. Talanta, 69, 456-462. https://doi.org/10.1016/j.talanta.2005.10.017

[22] Yuan, Y., Wang, Y., Liu, S., Li, Y., Duan, R., Zhang, H. and Hu, X. (2016) Fluorescence Quenching and Spectrophotometric Methods for the Determination of 6Mercaptopurine Based on Carbon dots. RSC Advances, 6, 52255-52263. https://doi.org/10.1039/C6RA07675K

[23] Li, Z., Wang, Y., Ni, Y.N. and Kokot, S. (2015) Fluorescence Analysis of 6-Mercaptopurine with the Use of a Nano-Composite Consisting of BSA-Capped Au Nano-Clusters and Core-Shell $\mathrm{Fe}_{3} \mathrm{O}_{4}-\mathrm{SiO}_{2}$ Nanoparticles. Biosensors and Bioelectronics, 70, 246-253. https://doi.org/10.1016/j.bios.2015.03.035

[24] Chen, Z., Zhang, G., Chen, X., Chen, J., Liu, J. and Yuan, H. (2013) A Fluorescence Switch Sensor for 6-Mercaptopurine Detection Based on Gold Nanoparticles Stabilized by Biomacromolecule. Biosensors and Bioelectronics, 41, 844-847. https://doi.org/10.1016/j.bios.2012.07.079

[25] Gao, M.X., Xu, J.L., Li, Y.F. and Huang, C.Z. (2013) A Rapid and Sensitive Spectrofluorometric Method for 6-Mercaptopurine Using CdTe Quantum Dots. Analytical Methods, 5, 673-677. https://doi.org/10.1039/C2AY25971K

[26] Li, Z., Ni, Y. and Kokot, S. (2015) A New Fluorescent Nitrogen-Doped Carbon Dot System Modified by the Fluorophore-Labeled ssDNA for the Analysis of 6-Mercaptopurine and $\mathrm{Hg}(\mathrm{II})$. Biosensors and Bioelectronics, 74, 91-97. https://doi.org/10.1016/j.bios.2015.06.014

[27] Wang, L. and Zhang, Z.J. (2008) The Study of Oxidization Fluorescence Sensor with Molecular Imprinting Polymer and Its Application for 6-Mercaptopurine (6-MP) Determination. Talanta, 76, 768-771. https://doi.org/10.1016/j.talanta.2008.04.024

[28] Choi, J.Y., Kim, G.H., Guo, Z.Q., Lee, H.Y., Swamy, K.M.K., Pai, J., Shin, S., Shin, I. and Yoon, J. (2013) Highly Selective Ratiometric Fluorescent Probe for $\mathrm{Au}^{3+}$ and Its Application to Bioimaging. Biosensors and Bioelectronics, 49, 438-441. https://doi.org/10.1016/j.bios.2013.05.033

[29] Lei, Y.J., Xue, C., Zhang, S.C. and Sha, Y.W. (2016) A Ratiometric Fluorescent Probe for Sensing Hydrogen Peroxide Based on a Hemicyanine-Naphthol Fluorophore. Luminescence, 31, 660-664. https://doi.org/10.1002/bio.3008

[30] Zhao, A.D., Chen, Z.W., Zhao, C.Q., Gao, N., Ren, J.S. and Qu, X.G. (2015) Recent Advances in Bioapplications of C-Dots. Carbon, 85, 309-327. https://doi.org/10.1016/j.carbon.2014.12.045

[31] Zheng, Y.K., Lai, L.M., Liu, W.W., Jiang, H. and Wang, X.M. (2017) Recent Advances in Biomedical Applications of Fluorescent Gold Nanoclusters. Advances in Colloid and Interface Science, 242, 1-16. https://doi.org/10.1016/j.cis.2017.02.005 
[32] Ren, X.L., Ge, J.J., Li, S., Shao, H.B., Qiu, X.Z., Tang, F.Q. and Meng, X.W. (2017) A Dual-Emission Nanohybrid of Gold Nanoclusters and Carbon Dots for Ratiometric Fluorescence Detection of Reactive Oxygen Species and Glucose. Journal of Biomedical Nanotechnology, 13, 1425-1434. https://doi.org/10.1166/jbn.2017.2432

[33] Ju, E.G., Liu, Z., Du, Y.D., Tao, Y., Ren, J.S. and Qu, X.G. (2014) Heterogeneous Assembled Nanocomplexes for Ratiometric Detection of Highly Reactive Oxygen Species in Vitro and in Vivo. Acs Nano, 8, 6014-6023. https://doi.org/10.1021/nn501135m

[34] Niu, W.J., Shan, D., Zhu, R.H., Deng, S.Y., Cosnier, S. and Zhang, X.J. (2016) Dumbbell-Shaped Carbon Quantum dots/AuNCs Nanohybrid as an Efficient Ratiometric Fluorescent Probe for Sensing Cadmium(II) Ions and L-Ascorbic Acid. Carbon, 96, 1034-1042. https://doi.org/10.1016/j.carbon.2015.10.051

[35] Li, Z.H., Guo, S., Yuan, Z.Q. and Lu, C. (2017) Carbon Quantum Dot-Gold Nanocluster nanoSatellite for Ratiometric Fluorescence Probe and Imaging for Hydrogen Peroxide in Living Cells. Sensors and Actuators B: Chemical, 241, 821-827. https://doi.org/10.1016/j.snb.2016.10.134

[36] Xie, H.Z., Dong, J., Duan, J.L., Waterhouse, G.I.N., Hou, J.Y. and Ai, S.Y. (2018) Visual and Ratiometric Fluorescence Detection of $\mathrm{Hg}^{2+}$ Based on a Dual-Emission Carbon Dots-Gold Nanoclusters Nanohybrid. Sensors and Actuators B: Chemical, 259, 1082-1089. https://doi.org/10.1016/j.snb.2017.12.149

[37] Liu, W., Wang, X.Y., Wang, Y.Q., Li, J.H., Shen, D.Z., Kang, Q. and Chen, L.X. (2018) Ratiometric Fluorescence Sensor Based on Dithiothreitol Modified Carbon Dots-Gold Nanoclusters for the Sensitive Detection of Mercury Ions in Water Samples. Sensors and Actuators B: Chemical, 262, 810-817. https://doi.org/10.1016/j.snb.2018.01.222

[38] Yan, Y.H., Yu, H., Zhang, K., Sun, M.T., Zhang, Y.J., Wang, X.K. and Wang, S.H. (2016) Dual-Emissive Nanohybrid of Carbon Dots and Gold Nanoclusters for Sensitive Determination of Mercuric Ions. Nano Research, 9, 2088-2096. https://doi.org/10.1007/s12274-016-1099-5

[39] He, Y.S., Pan, C.G., Cao, H.X., Yue, M.Z., Wang, L. and Liang, G.X. (2018) Highly Sensitive and Selective Dual-Emission Ratiometric Fluorescence Detection of Dopamine Based on Carbon Dots-Gold Nanoclusters Hybrid. Sensors and Actuators B: Chemical, 265, 371-377. https://doi.org/10.1016/j.snb.2018.03.080

[40] Yu, X., Zhang, C.X., Zhang, L.N., Xue, Y.R., Li, H.W. and Wu, Y.Q. (2018) The Construction of a FRET Assembly by Using Gold Nanoclusters and Carbon Dots and Their Application as a Ratiometric Probe for Cysteine Detection. Sensors and Actuators B: Chemical, 263, 327-335. https://doi.org/10.1016/j.snb.2018.02.072

[41] Gui, R.J., He, W.J., Jin, H., Sun, J. and Wang, Y.F. (2018) DNA Assembly of Carbon Dots and 5-Fluorouracil Used for Room-Temperature Phosphorescence Turn-On Sensing of AFP and AFP-Triggered Simultaneous Release of Dual-Drug. Sensors and Actuators B: Chemical, 255, 1623-1630. https://doi.org/10.1016/j.snb.2017.08.178

[42] Xie, J.P., Zheng, Y.G. and Ying, J.Y. (2009) Protein-Directed Synthesis of Highly Fluorescent Gold Nanoclusters. Journal of the American Chemical Society, 131, 888-889. https://doi.org/10.1021/ja806804u

[43] Zhao, Q., Chen, S.N., Zhang, L.Y., Huang, H.W., Zeng, Y.L. and Liu, F.P. (2014) Multiplex Sensor for Detection of Different Metal Ions Based on On-Off of Fluorescent Gold Nanoclusters. Analytica Chimica Acta, 852, 236-243. https://doi.org/10.1016/j.aca.2014.09.029 
[44] Ding, H., Liang, C., Sun, K.B., Wang, H., Hiltunen, J.K., Chen, Z.J. and Shen, J.C. (2014) Dithiothreitol-Capped Fluorescent Gold Nanoclusters: An Efficient Probe for Detection of Copper(II) Ions in Aqueous Solution. Biosensors and Bioelectronics, 59, 216-220. https://doi.org/10.1016/j.bios.2014.03.045

[45] Bain, D., Maity, S., Paramanik, B. and Patra, A. (2018) Core-Size Dependent Fluorescent Gold Nanoclusters and Ultrasensitive Detection of $\mathrm{Pb}^{2+}$ Ion. ACS Sustainable Chemistry \& Engineering, 6, 2334-2343.

https://doi.org/10.1021/acssuschemeng.7b03794

[46] Zhang, H.Y., Liu, Q., Wang, T., Yun, Z.J., Li, G.L., Liu, J.Y. and Jiang, G.B. (2013) Facile Preparation of Glutathione-Stabilized Gold Nanoclusters for Selective Determination of Chromium(III) and Chromium(VI) in Environmental Water Samples. Analytica Chimica Acta, 770, 140-146.

https://doi.org/10.1016/j.aca.2013.01.042 